\title{
CD44s as a surrogate marker for distinguishing intraductal papilloma from papillary carcinoma of the breast
}

\author{
Maruf Saddik, Raymond Lai
}

\begin{abstract}
Background-It has been shown that CD44 variants are differentially expressed in normal and neoplastic breast tissues. The diagnostic value of these markers in distinguishing benign from malignant breast lesions has not been well examined. Aims - To evaluate the diagnostic value of CD44s in distinguishing between intraductal papillomas and papillary carcinomas of the breast, which may be difficult morphologically.

Methods-Expression of CD44s detected by immunohistochemistry was studied in a series of intraductal papillomas (11) and papillary carcinomas (10). The normal breast tissues surrounding the lesions of these cases served as a control. The number of CD44s positive epithelial cells was scored and categorised as $<10 \%$, $10-70 \%$, or $>70 \%$.

Results-Normal breast epithelial cells and all intraductal papillomas (11 of 11) expressed CD44s in a high proportion of cells $(>70 \%)$. In contrast, the majority of papillary carcinoma cases (eight of 10) expressed this marker in $<10 \%$ of the cells. In the remaining two papillary carcinoma cases, positivity was seen in more than $10 \%$ but still less than $70 \%$ of the cells.

Conclusions-CD44s detection by immunohistochemistry is useful in distinguishing intraductal papillomas from papillary carcinomas of the breast.

(f Clin Pathol 1999;52:862-864)
\end{abstract}

Keywords: CD44s; intraductal papilloma; papillary carcinoma of breast

Morphological distinction between intraductal papilloma and papillary carcinoma of the breast is usually possible. Microscopic features favouring papillary carcinomas over intraductal papillomas include the absence of myoepithelial cells or apocrine features, the presence of minimal stroma, nuclear atypia, a high mitotic rate, and uniformity of tumour cells. ${ }^{1}$ However, there are occasional lesions that have morphological features of both intraductal papillomas and papillary carcinomas. ${ }^{12}$ Moreover, intraductal papillomas may rarely develop malignant transformation. ${ }^{3}$ For these problematic cases, there is no established ancillary method that may help establish the correct diagnosis.

CD44 is a polymorphic family of cell surface proteoglycans and glycoproteins implicated in cell-cell and cell-matrix adhesion interactions, cell migration, and tumour metastasis. ${ }^{45}$ Various CD44 species have been shown to be differentially expressed in benign and malignant cells, and downregulation of CD44s is associated with malignant transformation in the breast. ${ }^{6}$ Previous studies have so far focused on exploring a potential prognostic role for CD44 isoforms in malignancy. ${ }^{7-10}$ However, the potential diagnostic value of this marker has received little attention.

In this study, we tested the hypothesis that detection of CD $44 \mathrm{~s}$ by immunohistochemistry is a useful method for distinguishing intraductal papillomas from papillary carcinomas.

\section{Methods}

TISSUES AND IMMUNOHISTOCHEMISTRY

Eleven cases of intraductal papilloma and 10 of papillary carcinoma were retrieved from the files of the department of pathology, Royal University Hospital, University of Saskatchewan, Canada. Among the 10 cases of papillary carcinoma, eight were in situ. The other two were associated with a significant invasive component. All tissues were formalin fixed, routinely processed, and paraffin embedded. The monoclonal antibody reactive with CD44s (clone F10-44-2, Novocastra/Vector Laboratories) was used at a dilution of 1:50. A heat induced antigen retrieval method was used. Briefly, sections were placed in $0.01 \mathrm{~mol} / 1$ citrate buffer at $\mathrm{pH} 6.0$ and heated twice in a microwave oven for 12 minutes. Subsequently, the sections were left in warm citrate buffer for an additional 12 minutes. Reactivity was detected by an avidinbiotin immunoperoxidase detection system using 3,3'-diaminobenzidine-tetrahydrochloride dihydrate (Ventana Biotek Medical System) as the chromogen.

SCORING OF POSITIVE CELLS

At least 200 epithelial cells were counted in the areas of the lesions which were evenly stained. Scoring was performed independently by both authors. On the basis of these results, three grades of positivity were defined: $<10 \%$ of cells, $10-70 \%$ of cells, and $>70 \%$ of cells.

\section{Results}

There was a general agreement in the assignment of grades between the two authors. The results are summarized in table 1 . All cases of intraductal papilloma (11 of 11) showed $>70 \%$ cells positive for CD44s (fig 1, A and B) This positivity was evenly distributed throughout the lesion. The reactivity was membranous and was generally restricted to the basal and 
Table 1 Expression of CD44s in normal breast tissue, intraduct papilloma, and papillary carcinoma

\begin{tabular}{llll}
\hline Lesion & $<10 \%$ & $10-70 \%$ & $>70 \%$ \\
\hline Normal breast tissue $(\mathrm{n}=21)$ & 0 & 0 & 21 \\
Intraduct papilloma $(\mathrm{n}=11)$ & 0 & 0 & 11 \\
Papillary carcinoma $(\mathrm{n}=10)$ & 8 & 2 & 0
\end{tabular}

basolateral aspects of the epithelial cells. Myoepithelial cells were also positive. In contrast, most cases of papillary carcinomas (eight of 10 ) had $<10 \%$ cells positive for CD44s; four of these cases were entirely negative (fig 1, C and D). The other four cases had occasional positive cells with a total positivity of $<10 \%$. The remaining two cases showed $10-70 \%$ positivity. The staining pattern in papillary carcinomas cases that expressed CD44s was most striking in the basal and basolateral cell membranes.

Benign breast tissues adjacent to the lesions of these same cases $(n=21)$ were also assessed. As with intraductal papillomas, the normal epithelial cells in these tissues showed strong and diffuse CD 44 s reactivity at their basolateral (but not the luminal) surfaces. The stroma was focally positive; the positivity involved the leucocytes and occasional fibroblasts as well as blood vessels. Similar stromal

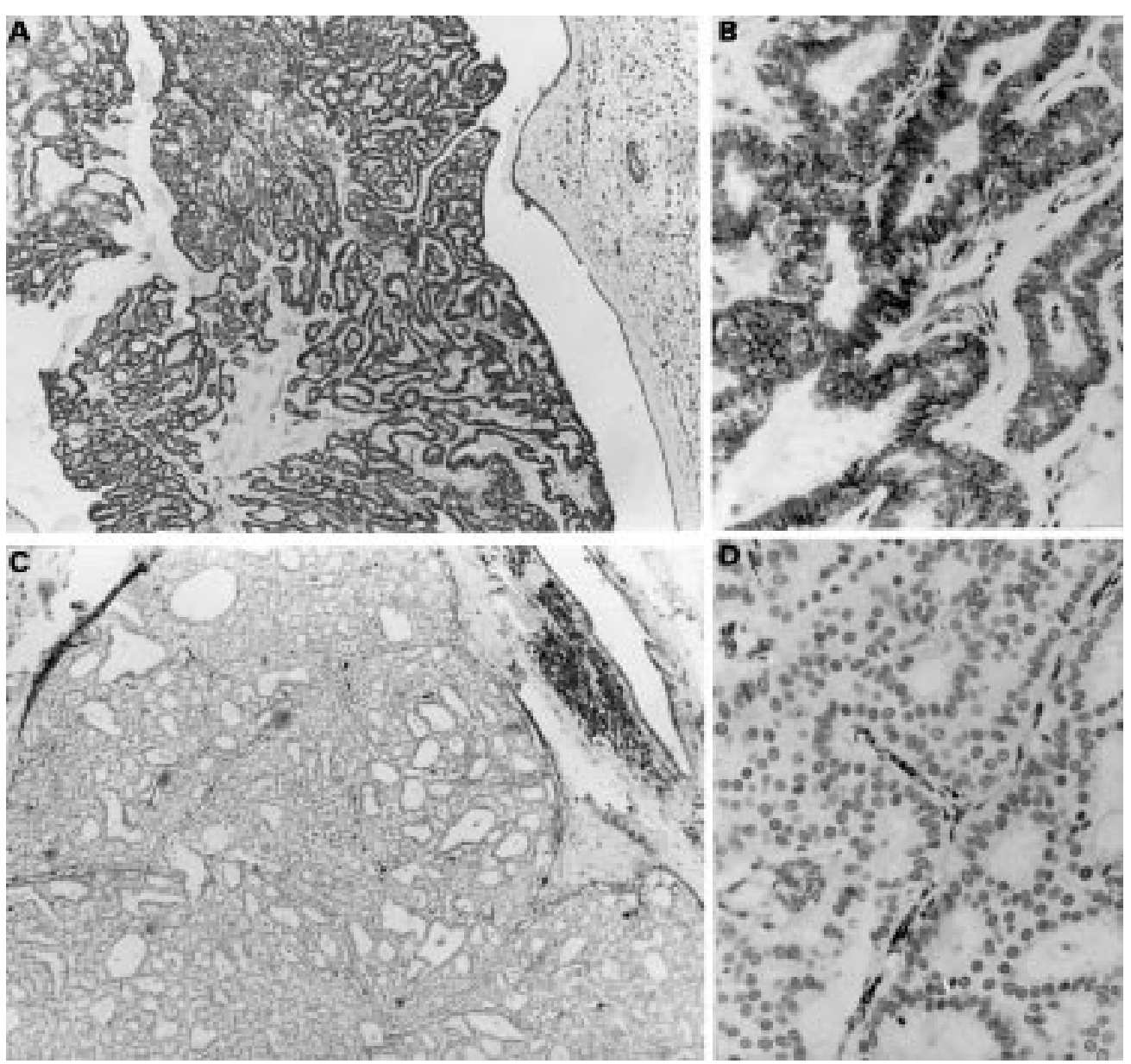

staining pattern was seen in intraductal papillomas and papillary carcinomas.

\section{Discussion}

The morphological distinction between intraductal papillomas and papillary carcinomas of the breast can sometimes be difficult. Immunodetection of smooth muscle actin ${ }^{11}$ and carcinoembryonic antigen (CEA) ${ }^{12}$ has been tested in this setting. However, these are not very useful. In a few previous studies it has been shown that various CD44 species are differentially expressed in normal and neoplastic breast tissues. For instance, expression of CD $44 v 6$ is upregulated in a subset of invasive carcinoma of the breast, and the phenotype correlates with a longer overall survival of the patients. On the other hand, the expression of CD44s is more commonly found in benign/hyperplastic breast tissues than in malignant breast lesions. Because of the latter finding, we hypothesised that CD44s may be a useful marker to distinguish intraductal papillomas from papillary carcinomas.

As shown in this study, the expression of CD 44 s in intraductal papillomas was distinctly different from that of papillary carcinomas. Using a positivity of $>70 \%$ as the cut off point,

Figure 1 Immunohistochemical staining for CD44s in intraductal papillomas and papillary carcinomas

(immunoperoxidase method). (A) CD44s expression in intraductal papilloma. Note the strong and diffuse staining pattern $(\times 31)$. (B) A high power view of the same lesion, showing the diffuse expression of this protein within the basal and basolateral cell membranes $(\times 311)$. (C) CD44s expression in papillary carcinoma. Note the total negative staining pattern for this protein (×31). (D) A high power view of the same lesion. Only stromal cells stain positive for this marker. The epithelial tumour cells are exclusively negative $(\times 311)$. 
papillary carcinomas cases can be separated from intraductal papillomas. While two cases of papillary carcinoma showed moderate positivity, even so less than $70 \%$ of the cells were positive. Furthermore, our data clearly show that the majority of papillary carcinoma cases $(80 \%)$ show a low positivity that is easily discernible from the high positivity seen in intraductal papillomas. Therefore immunostaining for CD44s is clearly a better marker to distinguish between these two lesions than those described previously.

These results support the view that immunodetection of CD44s is a useful method in distinguishing intraductal papillomas from papillary carcinomas. In problematic cases, this method may help to establish the correct diagnosis. However, a major limitation of the current study is the small number of cases examined. In addition, borderline papillary lesions were not evaluated. Therefore additional studies are needed to evaluate the expression of this marker in a larger number of cases, including the difficult borderline papillary lesions.
1 Rosen PP, ed. Papillary carcinoma. In: Rosen's breast patholgy. Philadelphia: Lippincott-Raven, 1997:335-54.

2 Kraus FT, Neubecker RD. The differential diagnosis of papillary tumors of the breast. Cancer 1962;5:444-55.

3 Rosen PP, Oberman HA. Invasive carcinoma. In: Atlas of tumor pathology: tumors of the mammary gland. Washington DC: Armed Forces Institute of Pathology, 1992:207-19.

4 Price EA, Coombe DR, Murray JC. Endothelial CD44H mediates adhesion of a melanoma cell line to quiescent mediates adhesion of a melanoma cell line to quiescent
human endothelial cells in vitro. Int 7 Cancer 1996;65:513human.

5 Gunthert U. CD44 in malignant disorders. Curr Topics Microbiol Immunol 1993;184:271-85.

6 Bankfalvi A, Terpe HJ, Breukelmann D, et al. Gains and losses of CD44 expression during breast carcinogenesis and tumor progression. Histopathology 1998;33:107-16.

7 Regidor PA, Callies R, Regidor M, et al. Expression of the CD44 variant isoforms 6 and $4 / 5$ in breast cancer. Correlation with established prognostic parameters. Arch Gynecol Obstet 1996;258:125-35.

8 Guriec N, Marcellin L, Gairard B, et al. CD44 exon 6 expression as a possible early prognostic factor in primary node negative breast carcinoma. Clin Exp Metastasis 1996; $14: 434$.

9 Schumacher U, Horny HP, Horst HA, et al. A CD44 variant exon 6 epitope as a prognostic indicator in breast cancer. Eur F Surg Oncol 1996;22:259-61.

10 Friedrichs K, Franke F, Lisboa B-W, et al. CD44 isoforms correlate with cellular differentiation but not with prognosis in human breast cancer. Cancer Res 1995;55:5424-33.

11 Murad TM, Swaid S, Pritchett P. Malignant and benign papillary lesions of the breast. Hum Pathol 1977;8:379-90.

12 Papotti M, Gugliotta P, Eusebi V, et al. Immunohistochemical analysis of benign and malignant papillary lesions of the breast. Am F Surg Pathol 1983;7:451-61. 